\title{
OMNI-DIRECTIONAL, SPECTRALLY SELECTIVE, WAFER-SCALE FABRICATED METALLIC-DIELECTRIC 2-D PHOTONIC CRYSTALS FOR HIGH TEMPERATURE ENERGY CONVERSION
}

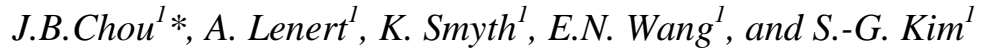 \\ ${ }^{1}$ Massachusetts Institute of Technology, Cambridge, Massachusetts, USA
}

\begin{abstract}
Two-dimensional metallic-dielectric photonic crystals $(\mathrm{MDPhCs})$ as spectrally selective absorbers and emitters are a promising technology for efficient heat-to-electricity energy conversion in solar thermophotovoltaic (STPV) systems [1]-[4]. Previously demonstrated metallic air photonic crystals (MAPhCs) are only able to absorb incident light at $\pm 30^{\circ}$ to normal, and had non-CMOS/MEMS compatible fabrication [5]. Here, we present a wafer-scale fabricated selective absorber and emitter which are stable at high temperatures $\left(1,000^{\circ} \mathrm{C}\right)$, possess omni-directional absorption measured up to $70^{\circ}$, contain tunable spectral control of absorption, and are polarization insensitive.
\end{abstract}

\section{INTRODUCTION}

One of the critical problems of traditional photovoltaic systems is their inability to efficiently store converted solar energy at low costs. The intermittent nature of solar energy renders photovoltaics incompatible with large scale grid level energy storage. However, STPV systems convert solar energy into thermal energy by means of a selective absorber where it can potentially be stored both efficiently and at low costs [6], [7]. When the energy is needed, the STPV system can then convert the thermal energy into electricity by means of a selective emitter and a photovoltaic device.

At the center of an STPV system lays the need for an efficient and robust selective absorber and emitter. Temperatures of STPV systems can reach as high as $1,000^{\circ} \mathrm{C}$ under concentrated solar energy. Previously demonstrated MAPhCs can serve as both selective absorbers and emitters and are able to survive high temperatures [2], [5], [8]. However, their limited angular absorption and non MEMS compatible fabrication techniques limit their performance and scalability. Plasmonic and metamaterial absorbers have yet to demonstrate high temperature stability and wafer-scale fabrication [9]-[12]. Thus, the MDPhC was originally developed to increase the high temperature stability of metallic nanostructures [13]. Here, we present a wafer-scale fabricated MDPhC by utilizing the sidewall lithography technique as shown in Fig. 1 [14]. The design, analysis, and fabrication of the MDPhC is presented. Measured Fourier transform infrared (FTIR) reflection spectrums demonstrate the wide-angle absorption. Scanning electron microscope (SEM) images demonstrate the robustness of the $\mathrm{MDPhC}$ after a high temperature exposure at $1,000^{\circ} \mathrm{C}$ for 24 hours.

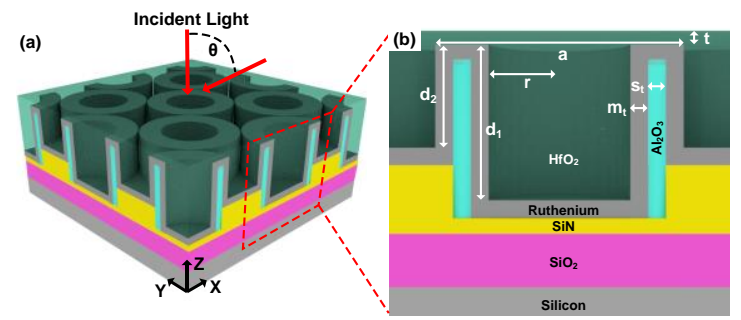

Figure 1: (a) Schematic of the MDPhC. (b) Cross-sectional schematic with period a, radius $r$, depths $d_{1}, d_{2}$, metal thickness $m_{t}$, $\mathrm{Al}_{2} \mathrm{O}_{3}$ thickness $\mathrm{s}_{t}$, and top dielectric thickness $t$.

\section{DESIGN}

The MDPhC utilizes cylindrical cavity modes to define the absorption spectrum and cutoff wavelength $\lambda_{c}$ [1], [15]. For incident light with wavelength $\left\langle\lambda_{c}\right.$, light is coupled to the cavity modes where the increased lifetime allows for the light to be absorbed by the metal. For light with wavelength $>\lambda_{c}$, light is not allowed to couple into the cavity modes and is reflected away. According to Kirchoff's law, under thermal equilibrium the absorption spectrum is equivalent to the emission spectrum, and thus the MDPhC is capable of tuning both absorption and emission. By tuning the radius $r$ and depths $d_{l}$ and $d_{2}$, the cutoff wavelength $\lambda_{c}$ can be spectrally tuned. Optimal absorption of the cavity modes can be determined via Q-matching [16].

The spectral tuning of the cut-off wavelength $\lambda_{c}$ is important for an efficient STPV system. A selective absorber optimally converts solar energy into heat by minimizing thermal radiative losses at wavelengths above the cutoff wavelength $\lambda_{c}$. A selective emitter optimally radiates towards a photovoltaic with a bandgap wavelength of $\lambda_{g}$. By tuning the cutoff wavelength to be equal to the bandgap, $\lambda_{c}=\lambda_{g}$, radiation above the bandgap wavelength, which cannot be converted into electron-hole pairs, is suppressed.

The omni-directional absorption is achieved with the dielectric filling inside the cavities which eliminates diffraction losses at oblique angles. The cavity modes can be tuned by the index $n$ of the dielectric inside the cavity, wherein the cutoff wavelength scales with $n$. By shifting the cutoff wavelength beyond the period of the structure, diffraction grating losses no longer apply which allows for wide angle absorption [1].

\section{FABRICATION}

In our wafer-scale fabrication process shown in Figure 2, a 6" silicon wafer is first deposited with a $250 \mathrm{~nm}$ thick diffusion barrier composed of $\mathrm{SiN}$ and thermal $\mathrm{SiO}_{2}$ to prevent the metal from diffusing into the silicon substrate at high temperatures. A $500 \mathrm{~nm}$ thick layer of sacrificial polysilicion layer is deposited via low-pressure chemical vapor deposition (LPCVD). A photolithographic stepper patterns resist on the polysilicon layer with an optimized checkerboard mask pattern to generate subwavelength gaps between the circular patterns, which is critical for optimal absorption in the MDPhCs. A sidewall lithography technique is used to define free-standing $\mathrm{Al}_{2} \mathrm{O}_{3}$ cylinders with a thickness of $40 \mathrm{~nm}$ [14], [17]-[19]. Ruthenium is then coated over the free-standing cylinders with conformal atomic layer deposition (ALD) with a thickness of $80 \mathrm{~nm}$. We have also experimentally verified that sputtered $\mathrm{W}$ had the same conformal deposition as $\mathrm{ALD}$, thus allowing for a wider variety of metals that can be successfully deposited at lower cost. A thick layer of $\mathrm{HfO}_{2}$ is deposited via ALD followed by a chemical mechanical polishing (CMP) step to remove the excess $\mathrm{HfO}_{2}$ and leave the exposed metallic-dielectric interface. SEM images of the final fabricated device as well as FIB created cross-section are shown in Figure 3. Due to slight variations across the large scale fabrication, the actual dimensions of the MDPhC unit cell can vary, thus the slight differences between images in Figure 3(a) and (b). 

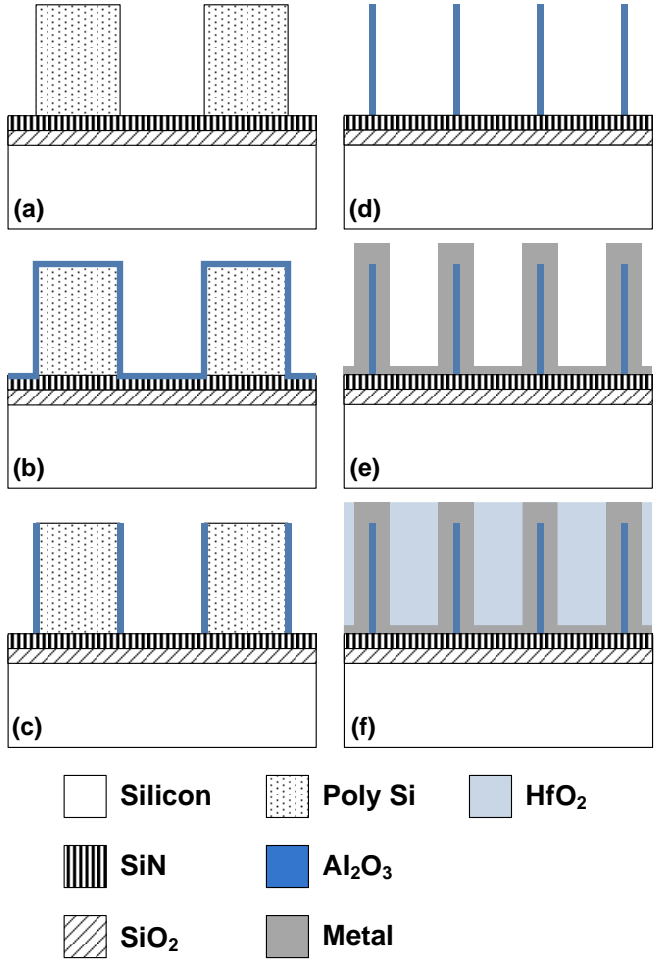

Figure 2: Schematic of the fabrication process. (a) Polysilicon layer is deposited and patterned on $\mathrm{SiN}$ and $\mathrm{SiO}_{2}$. (b) $A L D$ of $\mathrm{Al}_{2} \mathrm{O}_{3}$. (c) Anisotropic $\mathrm{Cl}_{2} \mathrm{RIE}$. (d) Removal of polysilicon with $\mathrm{XeF}_{2}$. (e) ALD of ruthenium. (f) ALD of $\mathrm{HfO}_{2}$ followed by $\mathrm{CMP}$ to expose metal dielectric interface.
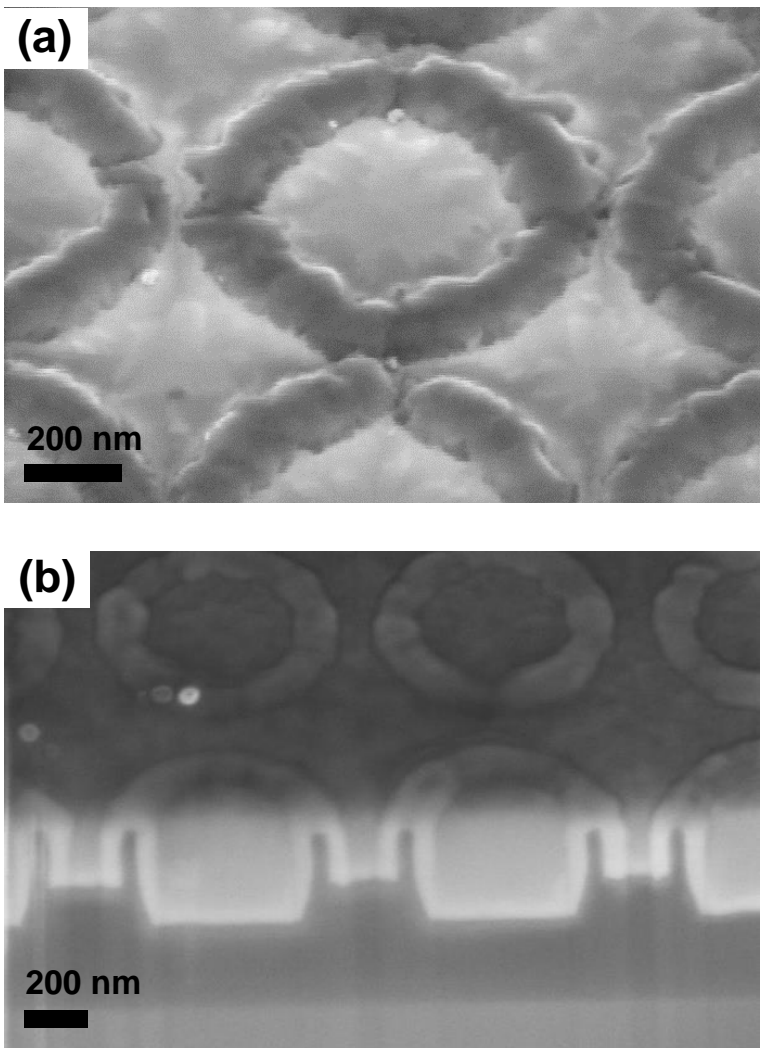

Figure 3: SEM images of the fully fabricated metallic dielectric photonic crystal: (a) top view and (b) angled cross-section view via FIB. The scale bars are $200 \mathrm{~nm}$. The measured values are $a=780 \mathrm{~nm}, r=600 \mathrm{~nm}, d_{1}=380 \mathrm{~nm}, d_{2}=200 \mathrm{~nm}, m_{t}=80 \mathrm{~nm}$, $s_{t}=40 \mathrm{~nm}$, and $t$ is estimated to be $t=25 \mathrm{~nm}$.

\section{RESULTS}

Reflection measurements, $R$, of the MDPhCs were performed in a Fourier transform infrared (FTIR) spectrometer. Subsequently, absorption is calculated as $\alpha_{s}=1-R-T$, where the transmission, $T$, is $T=0$ due to the thickness of the metal layer. Since we are measuring the reflection at wavelengths greater than the period, diffractive reflections are nonexistent [1]. The absolute absorption is measured in reference to a commercial aluminum reference mirror with known reflection spectrums at $30^{\circ}, 45^{\circ}, 50^{\circ}, 60^{\circ}$, and $70^{\circ}$.

The measured absorption spectrum of the MDPhC is shown in Figure 4(a) at angles up to $70^{\circ}$. Clearly the wavelength of the cutoff mode at $1.73 \mu \mathrm{m}$ does not change with incident angle, indicative of a flat dispersion curve of a cavity mode. The amplitude of the cut-off mode does decrease slightly with incident angle and is consistent with simulations [1]. Previously published work reported significant absorption decline for incident angles greater than $30^{\circ}$ due to diffraction losses [5]. Thus the improved angular performance of the MDPhC is experimentally confirmed. The distortions in the absorption spectra at approximately $\lambda=2.7$ $\mu \mathrm{m}$ and $\lambda=4.25 \mu \mathrm{m}$ are due to atmospheric absorptions. A slight absorption peak is observed at approximately $2.5 \mu \mathrm{m}$ and is due to the gap mode created between the metallic cylinders. Not all devices had the gap mode as fabrication variation caused only some devices to have an observable gap mode.
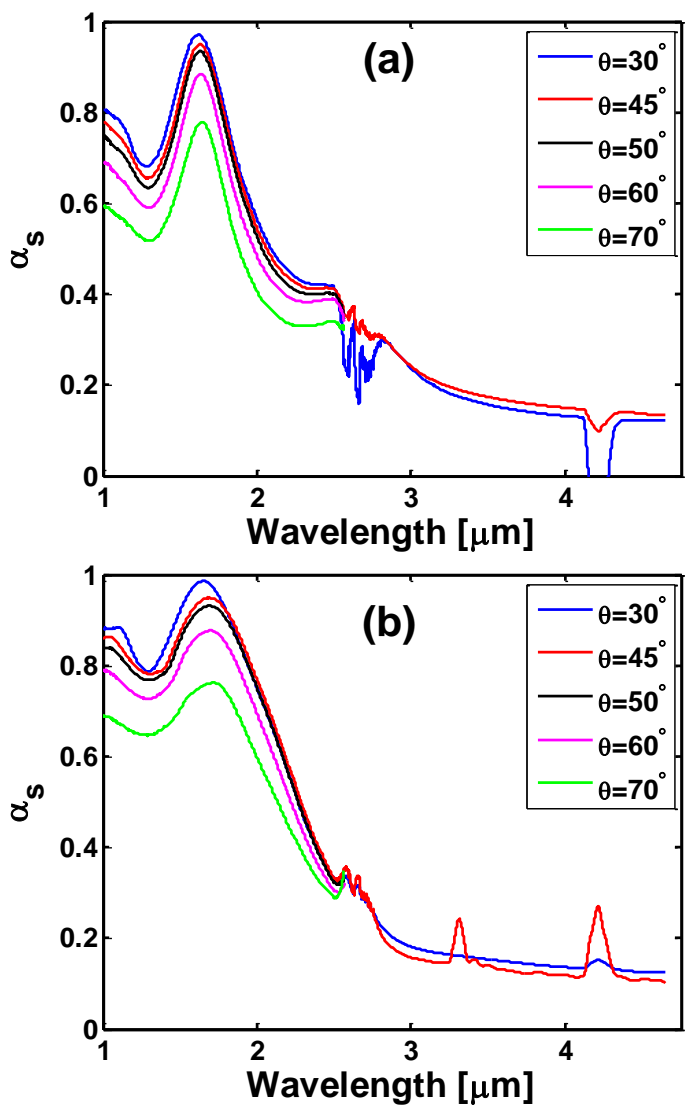

Figure 4: (a) Measured absorption spectrum of the MDPhC at various angles before the high temperature test. (b) Measured 
absorption spectrum after the high temperature test at $1,000^{\circ} \mathrm{C}$ for $24 \mathrm{hrs}$ in a $95 \% \mathrm{Ar}$ and $5 \% \mathrm{H}_{2}$ environment.

To verify the high temperature stability of the MDPhC structures, the samples were placed in a furnace at $1,000^{\circ} \mathrm{C}$ for 24 hours in a $95 \% \mathrm{Ar}$ and $5 \% \mathrm{H}_{2}$ environment to prevent oxidation. The measured absorption spectra of the same device shown in Figure 4(a) after the furnace test are shown in Figure 4(b). The linewidths of the cut-off mode have increased for the post-furnace samples, indicating to a lower Q mode. This may be attributed to annealing effects of the metal and dielectric or diffusion of the materials into each other. However, a larger Q smoothens out the absorption spectrum causing a more uniform absorption profile, although at the expense of a less steep cut-off. The wavelength of the cut-off mode remains relatively unchanged after the high temperature test which implies the structural integrity of the MDPhC is able to withstand the high temperature environment. The high temperature robustness is due to the $\mathrm{HfO}_{2}$ filling which minimizes surface diffusion [13].

SEM Images of various MDPhC samples after the high temperature tests are shown in Figure 5. The MDPhC sample measured in Figure 4 is shown in Figure 5(a), where the metal surface appears to have a layer of dielectric coating the surface, indicated by the lack of contrast between the rings and the dielectric area. In comparison to Figure 3(a), the metal and dielectric show a strong contrast due to the conductive nature of the metal. The coating may be due to oxidation of the metal or a reflow of the $\mathrm{HfO}_{2}$ over the metal surfaces. No physical damage to the $\mathrm{MDPhC}$ was observed for fully fabricated devices, which is significant due to the many different materials each with their own coefficients of thermal expansion.

Although no damage was observed within the MDPhC, outside of the MDPhC area crack and peeling effects were observed between the $\mathrm{HfO}_{2}$ layer and the substrate, as shown in Figure 5(b). The crack propagation outside of the MDPhC area never permeated the actual lattice and can be seen bending at a $90^{\circ}$ turn along the edge of the MDPhC. This shows the mechanical robustness of the MDPhC structure to high temperatures which is due to the high density of the periodic structure and the change of thickness in the MDPhC area.

For MDPhC structures with incomplete $\mathrm{HfO}_{2}$ filling, cracks within the MDPhC were observed to occur along the lattice of the filling holes, as shown in Figure 5(c). Whether the cracks only exist on the $\mathrm{HfO}_{2}$ layer or permeate further into the structure will require further investigation. Please note that due to the nonuniformity of the CMP process across a single wafer, a gradient of $\mathrm{HfO}_{2}$ thicknesses remains on the top surface of a portion of the fabricated chipset. The device shown in Figure 5(c) is a chip with a relatively thick layer of $\mathrm{HfO}_{2}$ remaining. Cracks were only observed in devices with thick $\mathrm{HfO}_{2}$ layers, indicating the importance of removing the top dielectric layer for high temperature stability.

To test the structural durability of the MDPhC's for high frequency temperature fluctuations, the MDPhC samples were placed in a solar concentrator system with on/off pulses of length 1 minute each for up to 10 iterations. The maximum temperature reached during the "on" pulse was $900^{\circ} \mathrm{C}$ measured with a bonded thermocouple. No damage was observed for any of the samples measured. Thus only the long 24 hour high temperature tests were observed to cause change in the MDPhC samples.
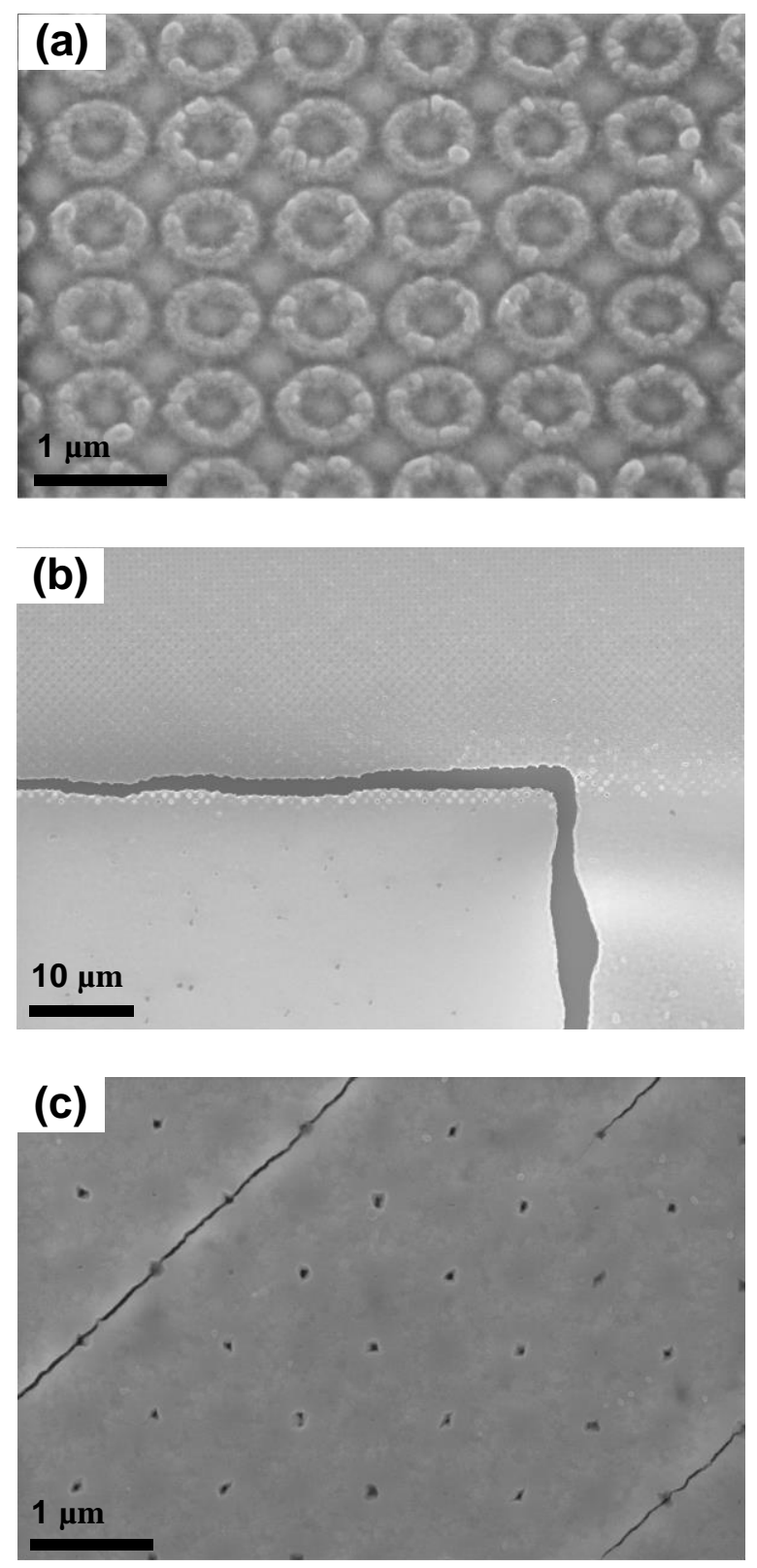

Figure 5: SEM images of the MDPhC after the high temperature tests. (a) Shows the sample measured in Figure 4 after the high temperature test. (b) The edge of the MDPhC is shown to be robust to crack propagation. (c) Inside the MDPhC lattice, cracks were only observed with samples with incomplete $\mathrm{HfO}_{2}$ filling. For devices with complete filling, no cracks were observed.

\section{CONCLUSION}

We demonstrate a high temperature stable wide-angle absorber utilizing dielectric filled cavity modes. A multi-layer wafer-scale fabrication process has been demonstrated which can lead to low-cost and massively scalable STPV systems. The fabrication process may also be extended into a double sided absorber/emitter chip for a compact and thermally efficient solar energy conversion system. Future work is still needed in regards to optical simulations, visible light absorption, and high temperature emittance measurements. We hope that the waferscale fabricated $\mathrm{MDPhC}$ will make large strides in the solar and thermal energy conversion space. 
This paper is based on the work supported as part of the Solid State Solar-Thermal Energy Conversion Center (S3TEC), an Energy Frontier Research Center funded by the U.S. Department of Energy, Office of Science, Office of Basic Energy Sciences under award DE-SC0001299/DE-FG02-09ER46577.

\section{REFERENCES}

[1] J. B. Chou, Y. X. Yeng, A. Lenert, V. Rinnerbauer, I. Celanovic, M. Soljačić, E. N. Wang, and S.-G. Kim, "Design of wide-angle selective absorbers/emitters with dielectric filled metallic photonic crystals for energy applications," Opt. Express, vol. 22, no. S1, pp. A144-A154, Jan. 2014.

[2] V. Rinnerbauer, S. Ndao, Y. Xiang Yeng, J. J. Senkevich, K. F. Jensen, J. D. Joannopoulos, M. Soljačić, I. Celanovic, and R. D. Geil, "Large-area fabrication of high aspect ratio tantalum photonic crystals for high-temperature selective emitters," J. Vac. Sci. Technol. B Microelectron. Nanometer Struct., vol. 31, no. 1, pp. $011802-011802-7$, Jan. 2013.

[3] A. Lenert, D. M. Bierman, Y. Nam, W. R. Chan, I. Celanović, M. Soljačić, and E. N. Wang, "A nanophotonic solar thermophotovoltaic device," Nat. Nanotechnol., vol. 9, no. 2, pp. 126-130, Feb. 2014.

[4] J. B. Chou, K. Smyth, and S.-G. Kim, "Low aspect ratio nanophotonic filled cavities with Q-matching for scalable thermophotovoltaic power conversion," in 2013 IEEE Photonics Conference (IPC), 2013, pp. 576-577.

[5] Y. X. Yeng, M. Ghebrebrhan, P. Bermel, W. R. Chan, J. D. Joannopoulos, M. Soljačić, and I. Celanovic, "Enabling hightemperature nanophotonics for energy applications," Proc. Natl. Acad. Sci., vol. 109, no. 7, pp. 2280-2285, Feb. 2012.

[6] W. R. Chan, P. Bermel, R. C. N. Pilawa-Podgurski, C. H. Marton, K. F. Jensen, J. J. Senkevich, J. D. Joannopoulos, M. Soljačić, and I. Celanovic, "Toward high-energy-density, high-efficiency, and moderate-temperature chip-scale thermophotovoltaics," Proc. Natl. Acad. Sci., vol. 110, no. 14, pp. 5309-5314, Apr. 2013.

[7] A. Datas, D. L. Chubb, and A. Veeraragavan, "Steady state analysis of a storage integrated solar thermophotovoltaic (SISTPV) system," Sol. Energy, vol. 96, pp. 33-45, Oct. 2013.

[8] H. Sai, H. Yugami, Y. Kanamori, and K. Hane, "Solar selective absorbers based on two-dimensional W surface gratings with submicron periods for high-temperature photothermal conversion," Sol. Energy Mater. Sol. Cells, vol. 79, no. 1, pp. 35-49, Aug. 2003.

[9] Y. Cui, K. H. Fung, J. Xu, H. Ma, Y. Jin, S. He, and N. X. Fang, "Ultrabroadband Light Absorption by a Sawtooth Anisotropic Metamaterial Slab," Nano Lett., vol. 12, no. 3, pp. 1443-1447, Mar. 2012.

[10] C. Wu, B. Neuner, G. Shvets, J. John, A. Milder, B. Zollars, and S. Savoy, "Large-area wide-angle spectrally selective plasmonic absorber," Phys. Rev. B, vol. 84, no. 7, Aug. 2011.

[11] T. V. Teperik, F. J. García de Abajo, A. G. Borisov, M. Abdelsalam, P. N. Bartlett, Y. Sugawara, and J. J. Baumberg, "Omnidirectional absorption in nanostructured metal surfaces," Nat. Photonics, vol. 2, no. 5, pp. 299-301, May 2008.

[12] N. P. Sergeant, O. Pincon, M. Agrawal, and P. Peumans, "Design of wide-angle solar-selective absorbers using aperiodic metal-dielectric stacks," Opt. Express, vol. 17, no. 25, p. 22800, Nov. 2009.

[13] H.-J. Lee, K. Smyth, S. Bathurst, J. Chou, M. Ghebrebrhan, J. Joannopoulos, N. Saka, and S.-G. Kim, "Hafnia-plugged microcavities for thermal stability of selective emitters,"
Appl. Phys. Lett., vol. 102, no. 24, pp. 241904-241904-5, Jun. 2013.

[14] T. J. Seok, A. Jamshidi, M. Eggleston, and M. C. Wu, "Massproducible and efficient optical antennas with CMOSfabricated nanometer-scale gap," Opt. Express, vol. 21, no. 14, pp. 16561-16569, Jul. 2013.

[15] J. D. Jackson, Classical Electrodynamics Third Edition, 3rd ed. Wiley, 1998.

[16] M. Ghebrebrhan, P. Bermel, Y. X. Yeng, I. Celanovic, M. Soljačić, and J. D. Joannopoulos, "Tailoring thermal emission via Q matching of photonic crystal resonances," Phys. Rev. A, vol. 83, no. 3, p. 033810, Mar. 2011.

[17] Y.-K. Choi, J. S. Lee, J. Zhu, G. A. Somorjai, L. P. Lee, and J. Bokor, "Sublithographic nanofabrication technology for nanocatalysts and DNA chips," J. Vac. Sci. Technol. B Microelectron. Nanometer Struct., vol. 21, no. 6, pp. 29512955, 2003.

[18] D. C. Flanders and N. N. Efremow, "Generation of [lessthan] $50 \mathrm{~nm}$ period gratings using edge defined techniques," J. Vac. Sci. Technol. B Microelectron. Nanometer Struct., vol. 1, no. 4, pp. 1105-1108, 1983.

[19] X. Liu, X. Deng, P. Sciortino, M. Buonanno, F. Walters, R. Varghese, J. Bacon, L. Chen, N. O'Brie, and J. J. Wang, "Large Area, $38 \mathrm{~nm}$ Half-Pitch Grating Fabrication by Using Atomic Spacer Lithography from Aluminum Wire Grids," Nano Lett., vol. 6, no. 12, pp. 2723-2727, Dec. 2006.

\section{CONTACT}

*J.B. Chou, jeffchou@mit.edu 